\title{
Utilisation de la photogrammétrie aérienne pour les relevés traditionnels de fluctuations des langues glaciaires
}

\author{
The use of aerial photogrammetry \\ for traditional surveys of fluctuations in glacial spits
}

R. Burnet

\author{
Division Glaciologie du CEMAGREF \\ Saint-Martin-d'Hères
}

Sous ce même titre, les 17 et 18 mars 1976, Messieurs Mura et Memier vous présentaient les motivations et l'intérêt d'une surveillance photogrammétrique de Glaciers Français menée par la Division Nivologie du CEMAGREF (ex-CTGREF). A cette date, nous étions dans la phase de mise en place du dispositif : huit ans plus tard, nous sommes dans la phase d'exploitation normale : un bref compte rendu des travaux réalisés et des documents disponibles est souhaitable; c'est l'objet de cette communication.

\section{Rappel du principe de surveillance photogrammétrique}

Le dispositif de surveillance est constitué :

- par une stéréopréparation constituée d'un réseau de repères visibles sur les photos aériennes dont les coordonnées doivent être connues dans le système Lambert et les altitudes rattachées au N.G.F.;

- par une prise de vue aérienne à une altitude moyenne de $1500 \mathrm{~m}$ au-dessus du sol, à l'exclusion de toute restitution photogrammétrique. ciers :

Ce dispositif est en place pour trois massifs de gla-

\section{Massif du Mont Blanc :}

. Glacier du Tour

- Glacier d'Argentière

- Mer de Glace

. Bossons

- Taconnaz

. Bionnassay

. Tré-la-Tête.

\section{Massif de la Savoie:}

Glacier des Sources de I'Isère

- Glacier des Sources de l'Arc

- Glacier du Mulinet

. Glacier du Grand Méan

- Glacier des Evettes

- Glacier d'Arnes

. Glacier de Gébroulaz

3. Massif de l'Oisans - Grandes Rousses

- Glacier de Sarennes

- Glacier de la Selle

- Glacier Blanc

- Glacier Noir

- Glacier du Chardon

. Glacier de la Pilatte

Chaque année un massif est observé; un massif est donc observé tous les trois ans.

Travaux nécessaires pour une mission annuelle

Avant chaque prise de vue, les repères de stéréopréparation doivent être visités et restaurés si besoin est. Il est à noter que la forme primitive de ces repères : carré blanc sur carré noir, a été remplacée au profit d'une croix blanche sur un fond noir, ceci dans un but de fiabilité d'identification de l'image de ces repères sur les clichés.

Ce travail nécessite une semaine de travail pour trois équipes de deux.

On peut estimer que $60 \%$ des repères sont en état et ne sont pas remplacés.

Le passage de l'avion qui effectue les prises de vues a lieu dès que ce travail de vérification est terminé et que les conditions météo sont favorables. 
Etat des documents disponibles et de leurs exploitations connues

\begin{tabular}{|c|c|c|c|c|c|}
\hline \multicolumn{6}{|c|}{ Caractéristiques communes à toutes les missions } \\
\hline \multirow{2}{*}{ Massif } & \multirow{2}{*}{ Glaciers } & \multicolumn{2}{|c|}{ Caractéristiques prises de vues } & \multirow{2}{*}{ Observations } & \multirow{2}{*}{ Triangulation } \\
\hline & & bande de vol & altitude & & \\
\hline Mont Blanc & $\begin{array}{l}\text { Tour } \\
\text { Argentière } \\
\text { Mer de Glace } \\
\text { Bossons } \\
\text { Taconnaz } \\
\text { Bionnassay } \\
\text { Tré-la-Tête }\end{array}$ & $\begin{array}{l}1 \text { bande transversale } \\
2 \text { bandes longitudinales } \\
1 \text { bande transversale } \\
\text { commune } \\
1 \text { bande transversale } \\
1 \text { bande longitudinale }\end{array}$ & $\begin{array}{c}4000 \mathrm{~m} \\
3500-4200 \mathrm{~m} \\
3400-4000 \mathrm{~m} \\
3300 \mathrm{~m} \\
3300 \mathrm{~m} \\
3800 \mathrm{~m}\end{array}$ & $\begin{array}{l}\text { même axe de vol : } \\
\text { deux altitudes }\end{array}$ & $\begin{array}{l}\text { commencée en } 1976 \\
\text { complétée en } 1979 \\
\text { à partir des nouveaux } \\
\text { points géodésiques, } \\
\text { triangulés la même année } \\
\text { par I'I.G.N. }\end{array}$ \\
\hline Savoie & $\begin{array}{l}\text { Source de l'Isère } \\
\text { Source de l'Arc } \\
\text { Mulinet } \\
\text { Grand Méan } \\
\text { Evettes } \\
\text { Arnes } \\
\text { Gébroulaz } \\
\end{array}$ & $\begin{array}{l}1 \text { bande longitudinale } \\
2 \text { bandes de vol } \\
\text { parallèles } \\
\text { communes } \\
\text { aux } 4 \text { glaciers } \\
1 \text { bande longitudinale } \\
1 \text { bande longitudinale } \\
\end{array}$ & $\begin{array}{l}4500 \mathrm{~m} \\
4300 \mathrm{~m} \\
4700 \mathrm{~m} \\
4300 \mathrm{~m} \\
4200 \mathrm{~m}\end{array}$ & & $\begin{array}{l}\text { réalisée en } 1974 \\
\text { à partir de la triangulation } \\
\text { I.G.N. de } 1971\end{array}$ \\
\hline $\begin{array}{l}\text { Oisans } \\
\text { Grandes- } \\
\text { Rousses }\end{array}$ & $\begin{array}{l}\text { Sarennes } \\
\text { La Selle } \\
\text { Blanc } \\
\text { Noir } \\
\text { Chardon } \\
\text { Pilatte }\end{array}$ & $\begin{array}{l}1 \text { bande longitudinale } \\
1 \text { bande transversale } \\
2 \text { bandes de vol parallèles } \\
\text { communes aux } 2 \text { glaciers } \\
1 \text { bande de vol } \\
\text { commune }\end{array}$ & $\begin{array}{l}4500 \mathrm{~m} \\
4200 \mathrm{~m} \\
4500 \mathrm{~m} \\
4700 \mathrm{~m} \\
4300 \mathrm{~m}\end{array}$ & & $\begin{array}{l}\text { réalisée en } 1978 \text { à partir de la } \\
\text { triangulation IGN de } 1972 \\
\text { triangulation locale } \\
\text { réalisée en } 1975 \\
\text { rattachement au système } \\
\text { Lambert réalisé par I'I.G.N. } \\
\text { lors de la triangulation } \\
\text { de I'Oisans en } 1983\end{array}$ \\
\hline
\end{tabular}

\begin{tabular}{|c|c|c|c|c|c|}
\hline \multicolumn{6}{|c|}{ Caractéristiques propres à chaque mission } \\
\hline \multicolumn{6}{|c|}{ Massif du Mont Blanc } \\
\hline \multicolumn{2}{|c|}{ Année de prise de vue } & \multicolumn{3}{|c|}{ Caractéristiques de prises de vues } & \multirow{2}{*}{ Observations } \\
\hline prévue & réalisée & focale & chambre & nature cliché & \\
\hline 1976 & 10.10 .1976 & 100,65 & WILD RC 7 & $\begin{array}{l}\text { plaque de verre } \\
\qquad 14 \times 14\end{array}$ & $\begin{array}{l}\text { les bandes hautes de Mer de Glace } \\
\text { et Argentière n'ont pas été réalisées }\end{array}$ \\
\hline 1979 & 05.09.1979 & 152,89 & ZEISS RMK & film $23 \times 23$ & stéréopréparation vérifiée \\
\hline 1982 & 22.08 .1982 & 151,69 & WILD RC10 & film $23 \times 23$ & stéréopréparation vérifiée \\
\hline \multicolumn{6}{|c|}{ Massif de la Savoie } \\
\hline 1974 & 25.09.1975 & 100,65 & WILD RC7 & $\begin{array}{l}\text { plaque de verre } \\
\qquad 14 \times 14 \\
\text { cette année, } \\
\text { Rousses }\end{array}$ & $\begin{array}{l}\text { n'a pu être réalisée en } 1974 \\
\text { en raison de la venue précoce de la neige } \\
\text { réalisée avec le Massif Oisans-Grandes }\end{array}$ \\
\hline 1977 & 15.09 .1978 & 100,65 & WILD RC7 & $\begin{array}{l}\text { plaque de verre } \\
\qquad 14 \times 14\end{array}$ & $\begin{array}{l}\text { n'a pu être réalisée en } 1977 \\
\text { en raison de I'accumulation exception- } \\
\text { nelle de neige qui a empêché les travaux } \\
\text { de stéréopréparation, stéréopréparation } \\
\text { non vérifiée en } 1978\end{array}$ \\
\hline 1980 & 27.08.1981 & 151,69 & WILD RC10 & film $23 \times 23$ & $\begin{array}{l}\text { idem } \\
\text { stéréopréparation vérifiée }\end{array}$ \\
\hline 1983 & 13.09.1983 & 151,69 & WILD RC 10 & film $23 \times 23$ & stéréopréparation vérifiée \\
\hline \multicolumn{6}{|c|}{ Massif de I'Oisans-Grandes Rousses } \\
\hline 1975 & 25.09.1975 & 100,65 & WILD RC7 & plaque de verre & \\
\hline 1978 & 15.09.1977 & 100,65 & WILD RC7 & $\begin{array}{l}\text { plaque de verre } \\
14 \times 14\end{array}$ & stéréopréparation vérifiée \\
\hline 1981 & 27.08.1981 & 151,69 & WILD RC10 & film $23 \times 23$ & stéréopréparation non vérifiée \\
\hline 1984 & $?$ & $?$ & $?$ & $?$ & $?$ \\
\hline
\end{tabular}




\section{Massif du Mont Blanc}

1976 : Restitution partielle des fronts au stéréotope par le laboratoire de Glaciologie du C.N.R.S. (L.G.G.E.) pour les glaciers suivants : Argentière, Mer de Glace, Bossons, Tré-la-Tête.

1979 : Restitution par le L.G.G.E. au stéréotope de la Mer de Glace entière pour les bandes de Forbes; Articles : - Lliboutry, Reynaud, Journal de Glaciologie 1981

- Reynaud, Z.G.G. 1979.

1981 : Restitution des fronts.

1976 Mémoire diplôme d'Ingénieur de l'ENISAIS Strasbourg de Jean Verrier : Mer de Glace; restitution des profils et

1979 comparaison avec les levés de terrain réalisés par M. Chaumeton.

\section{Massif de la Savoie}

1975 Mémoire diplôme d’Ingénieur de l'E.S.G.T. Evry de Ch. Vincent "Photogrammétrie appliquée à l'étude des

1978 fluctuations glaciaires ".

1980 Restitution des fronts au L.G.G.E.

Gebroulaz : restitution des fronts; Article Reynaud, Vallon, Carle T.S.P.V. 1983.

\section{Massif de l'Oisans - Grandes Rousses}

1975 : Restitution à titre d'essai de la langue du Glacier Blanc par Sintegra Meylan au 1/2000.

1981 : Restitution complète du glacier de Sarennes et des rochers couverts en 1904 au 1/5000, par Sintegra Meylan.

1975

1978 Restitution des langues du Glacier Blanc et du Glacier Noir par le LGGE.

1981

M. DE $C R E ́ E Y$ demande qui sont les utilisateurs actuels de ces observations et quel est le gain de précision obtenu par rapport aux anciens types de données. Selon. M. BURNET, on doit atteindre une précision de 30 à $40 \mathrm{~cm}$; il ajoute que les chercheurs du Laboratoire de Glaciologie du C.N.R.S. sont les utilisateurs habituels de ces documents. II signale ensuite que I'I.G.N. compte faire une prise de vues aériennes au 1/60 000 de la Haute Savoie en 1984. A ce sujet, M. LeCordix signale les efforts de I'I.G.N. dans le domaine des mesures de volume du manteau neigeux, par le biais de l'utilisation d'émulsions appropriées. Cette prise de vue ayant lieu au printemps dans le but de restituer des volumes d'eau.

$M$. VALLA regrette qu'une telle entreprise ne puisse donner beaucoup d'informations sur l'état des glaciers.

M. REYNAUD, utilisateur assidu des photographies du CEMAGREF, est satisfait de la précision obtenue, il souhaiterait cependant que l'on puisse établir un meilleur lien entre les nouveaux repères et les anciens des Eaux et Forêts. C'est quelquefois difficile, sinon impossible, de retrouver les anciens repères, répond M. BURNET, il précise aussi, sur une intervention de M. LÉvY, que les repères actuels sont situés en dehors des glaciers.

M. ROBERT évoque la possibilité d'observations par satellite. M. LECORDIX signale que la précision actuelle de l'imagerie par satellite est limitée à quelques dizaines de mètres. insuffisante donc pour l'objectif recherché ici: II.G.N. est cependant en possession d'un certain nombre d'images des Alpes, du Canigou et de l'Himalaya, prises par Spacelab, susceptibles d'être utiles à des chercheurs.

M. REYNAUD voit surtout un développement de ces méthodes vers l'obtention de bilans glaciaires.

M. BURCHET demande s'il est envisagé une extension des mesures du CEMAGREF, sur les Pyrénées. Faute de moyen. c'est impossible répond $M$. BURNET: $M$. de CRECY s'interroge alors sur l'importance de l'effort financier nécessaire, et sur la possibilité de le poursuivre. M. Le Président, citant l'exemple des mesures hydrologiques, est conscient des difficultés rencontrées pour assurer la continuité de ces mesures. d'autant plus que le bénéfice à en tirer n'est pas toujours immédiat. c'est un effort pour l'avenir.

M. ROBERT suggère que soit examinée la possibilité que par une intervention la S.H.F. joue un rôle moteur en ce domaine.

M. Le Président remercie M. RoBert de cette suggestion et propose son aide à $\mathrm{M}$. de CRÊCY pour essayer de la mettre en œuvre. 


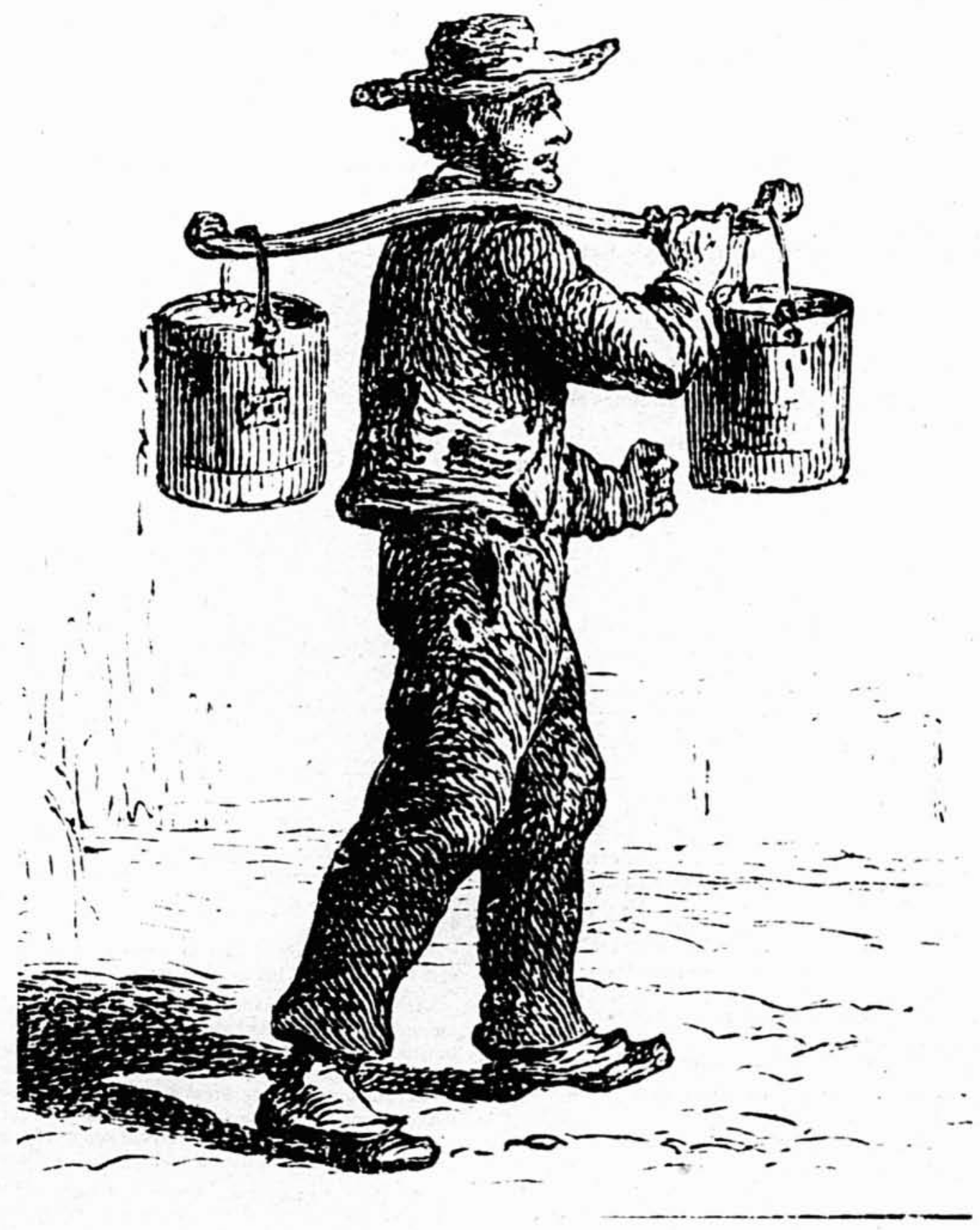

